\title{
Renal Cell Carcinoma and Mucinous Adenocarcinoma of Ureter after Renal Transplantation with Neoplasm of Urethra in Follow up
}

\author{
Caio Eduardo Gullo', João Paulo Pretti Fantin², Luis Cesar Fava \\ Spessoto ${ }^{3}$, Márcio Gatti ${ }^{3}$, José Germano Ferraz de Arruda ${ }^{3}$, Thiago \\ Antoniassi $^{3}$, Pedro Francisco Ferraz de Arruda ${ }^{3}$, Fernando Nestor \\ Facio Junior ${ }^{3}$
}

${ }^{1}$ Resident of the Cardiovascular Surgery Department - Hospital de Base/FAMERP
${ }^{2}$ Resident of the Urology Department - Hospital de Base/FAMERP
${ }^{3}$ Professor of the Surgical Specialties Department - Hospital de Base/FAMERP

Abstract: This paper presents the first case of a kidney graft recipient with double malignancy of the upper urinary system and cancer of the urethra evident four years after transplantation.

Keywords: kidney tumor, ureteral tumor, urethral cancer, kidney transplant

\section{INTRODUCTION}

Many patients are transplanted with unknown neoplasms. In the study by Denton et al., histopathologic evaluation of one of the primitive kidneys in a series of 260 patients identified 11 (4.2\%) cases of renal cell carcinoma (RCC); all of these patients were asymptomatic and the neoplasia had not been detected by imaging. Factors such as being male, recipient and donor age, recipient of African descent, prolonged dialysis, immunosuppressant therapy and acute post-transplant rejection are related to risk for RCC after renal transplant. ${ }^{2-4}$ This paper presents the first case of a renal transplant patient with double malignancy of the upper urinary system and cancer of the urethra evident after four years of follow-up.

\section{CASE REPORT}

A 62-year-old patient with nephrolithiasis had been monitored by the urology service of a university hospital since 2001. The patient progressed to endstage kidney disease (ESRD) with renal replacement therapy (RRT) being begun in February 2005. Early complications were observed after kidney transplantation in 2008, including delayed graft function and urinary tract infection (UTI). A computed tomography scan showed nephrolithiasis in the right kidney, a kidney stone at the ureteropelvic junction of the left kidney and hydronephrosis with a thick liquid content (Fig. 1).

Left nephrectomy was performed in 2008, with a marked amount of mucus secretion being observed in the renal pelvis and ureter lumen during the intraoperative period. A pathological examination of the kidney found a nodular lesion measuring $1.5 \mathrm{~cm}$ in diameter at the upper pole of the kidney (Fig. 2A) consisting of the clear neoplastic cells typical in RCC (Fig. 2B). There was also a very pale mass blocking the lumen of his ureter (Fig. 3C). Histologic analysis identified a primary papillary adenocarcinoma of a mucinous ureter associated with an epithelial metaplasia (Fig. 2D). In 2012, a follow-up urethrocystoscopy with biopsy after hematuria showed warty lesions in the penile urethra; the results of histological evaluation revealed well-differentiated and vegetating squamous cell carcinoma. After discussing the condition with the patient, and as the lesions were superficial and had been removed, the chosen conduct was active surveillance. Currently, 
the patient is asymptomatic and without further lesions.

\section{DISCUSSION}

The diagnosis is incidental in $30-60 \%$ of cases of RCC, ${ }^{6}$ especially in patients with ESRD, for whom the incidence of neoplasms and worse prognoses in terms of lesion grade and relapse are higher. ${ }^{7}$ No protective effect is observed with renal transplantation. ${ }^{8}$ At the end of the third postoperative year, there is a 15-fold higher risk of disease in transplant recipients compared to the general population in the United States (3.16 per 1000 person-years), and $39 \%$ higher than those on the waiting list for transplantation. ${ }^{5}$

Many patients are transplanted with unknown neoplasms. In the study by Denton et al., ${ }^{1}$ the histopathological evaluations of one of the primitive kidneys in a series of 260 patients identified 11 $(4.2 \%)$ cases of RCC; all of these patients were asymptomatic and the tumors were not identified by imaging before exeresis. Considering both organs, up to $8.4 \%$ of undiagnosed RCC are found at the time of renal transplantation; in this case, the incidental detection of RCC occurred five months after transplantation. One risk factor is acquired cystic kidney disease (ACKD), which consists of the formation of five or more cysts of $0.2-2.5 \mathrm{~cm}$ in the longest axis in kidneys without autosomal polycystic disease. $^{2}$

In the study by Suson et al., ${ }^{9} 23 \%$ of patients with ESRD had pre- or post-transplant cysts; in these cases, the cumulative incidence of RCC at 3 years post-transplantation was 9.29 per 1000 person-years $(2.3 \%)$, whereas in the absence of ESRD, the incidence was 3.08 per 1000 person-years $(0.7 \%)$. The higher occurrence and carcinogenicity of these cysts in ESRD suggests a pathological degeneration process triggered by the state of uremia, plus immunosuppression due to the underlying disease with consequent deficiency in immune surveillance, and the existence of nondialysable cancer-causing agents, which would justify their greater propensity to malignity and aggressiveness. ${ }^{10}$ Thus, the risk of death, which is already high for RCC diagnosed after renal transplantation, becomes considerably higher in patients with pre-transplant cysts.

Factors such as male gender, age of recipient and donor, recipient of African descent, prolonged dialysis, immunosuppressive therapy and acute posttransplant rejection are also reported as risk factors for renal transplant RCC. ${ }^{2-4}$ According to Suson et al., ${ }^{9}$ the cure rate for transplant patients with localized disease who undergo nephrectomy is high, with disease-related mortality being from $0-10 \%$. Thus, screening practices should prioritize the identification of high-risk patients, since the removal of the tumor in the native organ is potentially curative.

This patient also presented with a notable dilatation of the pyelocaliceal system due to an accumulation of mucus produced by a primary papillary adenocarcinoma of the ureter with a mucinous component. Thirteen cases of this neoplasm have been described in the renal pelvis or ureter. ${ }^{11,12}$ One case involved a horseshoe kidney, ${ }^{12}$ another a solitary pelvic kidney, ${ }^{11}$ and two cases were related to peritoneal pseudomyxoma. ${ }^{13}$ However, the description of a primary papillary adenocarcinoma associated with another oncologic lesion of the urinary system, a RCC in a kidney graft as reported in the current case, was not found in the literature.

The most common malignancies of the renal pelvis and ureter are of urothelial origin; squamous and glandular carcinomas that occur in areas of metaplastic transformation of that epithelium in response to long-term hydronephrosis, urinary tract infection or chronic urolithiasis are rare. ${ }^{14}$ The occurrence of kidney stones is very common in mucinous adenocarcinoma of the urinary tract, a condition that led some authors to postulate that glycoproteins secreted by tumor cells bind to cations, such as sodium, calcium and magnesium, to form large stones. ${ }^{15}$ Our patient evolved without a history of macroscopic or microscopic hematuria, and follow-up imaging did not detect malignancy.

In this case, the diagnosis of a neoplasia in the urethra is also rare after a 4-year follow-up, especially in male patients. ${ }^{16}$ The involvement of the penile urethra occurs in $30 \%$ of cases with symptomatology compatible with urethral stenosis, different from the current report. The prognosis is very variable, but 5 -year survival may reach $81 \%$ in tumors smaller than $2 \mathrm{~cm}$; in this report, the tumor was even smaller. ${ }^{17}$ The patient's choice of conduct was conservative and discordant with the literature. However, the evolution was satisfactory, and the polypoid lesions did not relapse after exeresis. Furthermore, no new lesions were evident after 4 years of follow up.

In summary, ESRD requires periodic evaluations of the primitive kidneys in both dialysis and transplant patients, with nephrectomy being a potentially curative modality for early-diagnosed RCC. Chronic cases of urolithiasis should be investigated for neoplasms triggered by this condition and secondary tumors. During the follow-up, any new symptoms should be investigated carefully because of the possibility of lesions not related to the initial tumor. 
Renal Cell Carcinoma and Mucinous Adenocarcinoma of Ureter after Renal Transplantation with Neoplasm of Urethra in Follow up

\section{REFERENCES}

1) Denton MD, Magee CC, Ovuworie C, Mauiyyedi S, Pascual M, Colvin RB, et al. Prevalence of renal cell carcinoma in patients with ESRD pre-transplantation: a pathologic analysis. Kidney Int. 2002;61(6):2201-2209.

2) Filocamo MT, Zanazzi M, Li Marzi V, Guidoni L, Villari D, Dattolo E, et al. Renal cell carcinoma of native kidney after renal transplantation: clinical relevance of early detection. Transplant Proc. 2009;41(10):4197-4201.

3) Kinsella SM, Coyle JP, Long EV, McWilliams SR, Maher MM, Clarkson MR, et al. Maintenance hemodialysis patients have high cumulative radiation exposure. Kidney Int. 2010;78:789-793.

4) Tsaur I, Karalis A, Probst M, Blaheta RA, Scheuermann EH, Gossmann J, et.al. Development of urological cancers in renal transplant recipients: 30-year experience at the Frankfurt Transplant Center. Cancer Sci. 2010;101(11):24302435.

5) Hurst FP, Jindal RM, Graham LJ, Falta EM, Elster EA, Stackhouse GB, et al. Incidence, Predictors, Costs, and Outcome of Renal Cell Carcinoma After Kidney Transplantation: USRDS Experience. Transplantation. 2010;90(8):898-904.

6) Pompeo ACL, Wroclawski EC, Sadi MV. Algoritmos em urooncologia. Rio de Janeiro: Elsevier, 2007.

7) Rao SN. Cancer screening in end-stage renal disease. Saudi J Kidney Dis Transpl. 2009;20:737-740.

8) Elkentaoui H, Robert G, Pasticier G, Bernhard JC, Couzi L, Merville $\mathrm{P}$, et al. Therapeutic management of de novo urological malignancy in renal transplant recipients: the experience of the French Department of Urology and Kidney
Transplantation from Bordeaux. Oncology. 2010;75(1):126132.

9) Suson KD, Sausville JE, Sener A, Phelan MW. Native nephrectomy for renal cell carcinoma in transplant recipients. Transplantation. 2011;27;91(12):1376-1379.

10) Goh A, Vathsala A. Native renal cysts and dialysis duration are risk factors for renal cell carcinoma in renal transplant recipients. Am J Transpl. 2011;11:86-92.

11) Wan J, Ohl DA, Weatherbee L. Primary mucinous adenocarcinoma of renal pelvis in solitary pelvic kidney. Urology. 1993;41(3):292-294.

12) Ueda T, Okumi M, Ichimaru $N$, Itoh $K$, Matsuoka $Y$, Fujimoto N. Mucinous adenocarcinoma of the renal pelvis in the horseshoe kidney: a case report. Hinyokika Kiyo. 2002;48(3):187-189.

13) Shah VB, Amonkar GP, Deshpande JR, Bhalekar $H$. Mucinous adenocarcinoma of the renal pelvis with pseudomyxoma peritonei. Indian $J$ Pathol Microbiol. 2008;51:536-537.

14) Kaur G, Naik VR, Rahman MNG. Mucinous adenocarcinoma of the renal pelvis associated with lithiasis and chronic gout. Singapore Med J. 2004;45(3):125-126.

15) Liwnicz BH, Lepow H, Schutte H, Fernandez R, Caberwal D. Mucinous adenocarcinoma of the renal pelvis: discussion of possible pathogenesis. J Urol. 1975;114:306-310.

16) Lutz ST, Huang DT. Combined chemoradiotherapy for locally advanced squamous cell carcinoma of the bulbomembranous urethra: a case report. $J$ Urol. 1995;153:1616-1618.

17) Walsh PC, Retik AB. Surgery of penile and urethral carcinoma. In: Campbell's Urology. 7th ed. WB Saunders, 1998:3395-3407.

Figure 1 - Computed tomography showing nephrolithiasis in the right and calculation in left ureteropelvic junction associated to exuberant hydronephrosis with thick liquid inside.

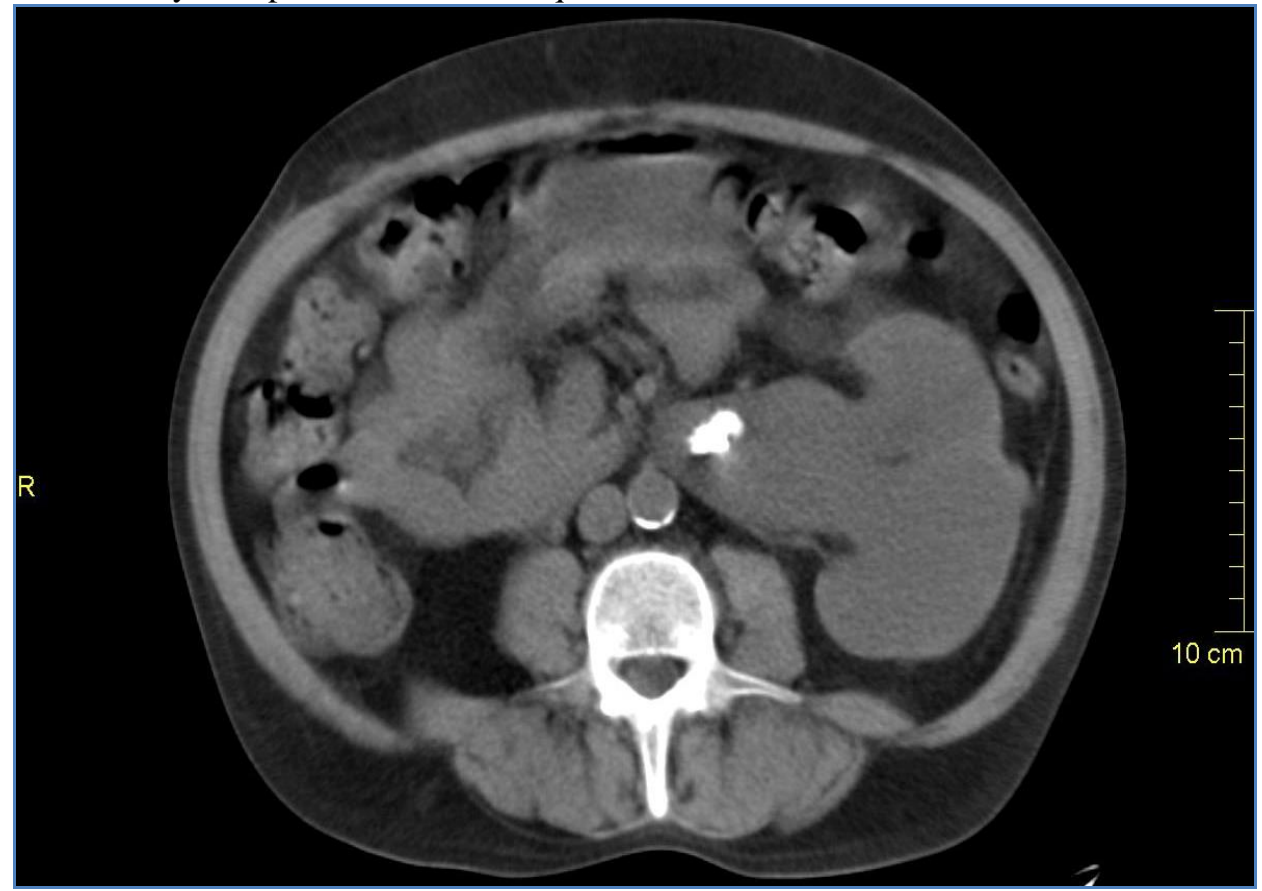


Figure 2 - (A) Nodular lesion measuring $1.5 \mathrm{~cm}$ in diameter in the upper pole of the left kidney. (B) Clear cell renal cell carcinoma does not extend to the adipose tissue of the renal sinus or the collecting system. (C) Lighter, solidlooking tissue in the left ureter, obstructing the lumen of the ureter. (D) Primary papillary adenocarcinoma of the ureter with mucinous component associated with epithelial metaplasia without invasion of the ureteral musculature.

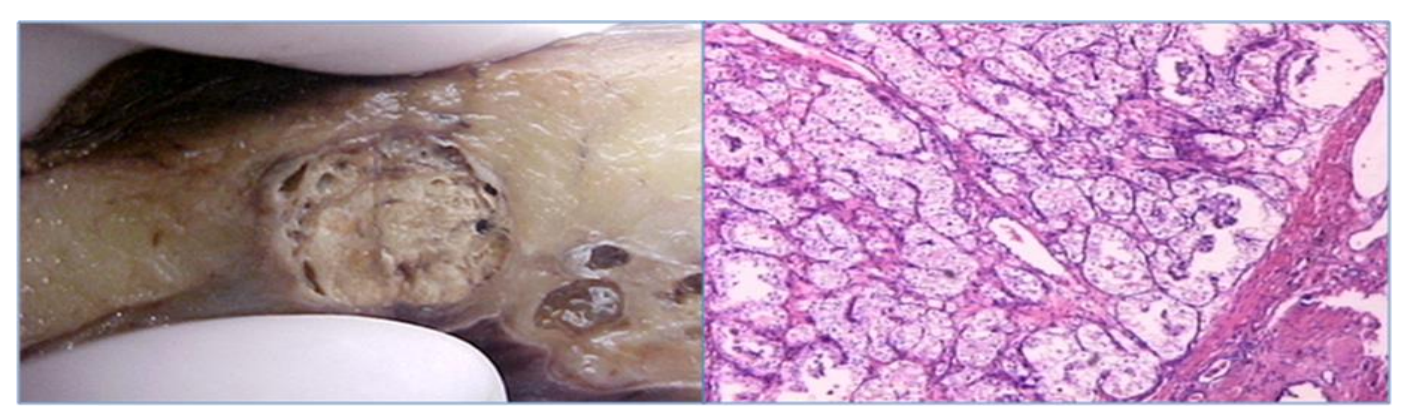

A

B

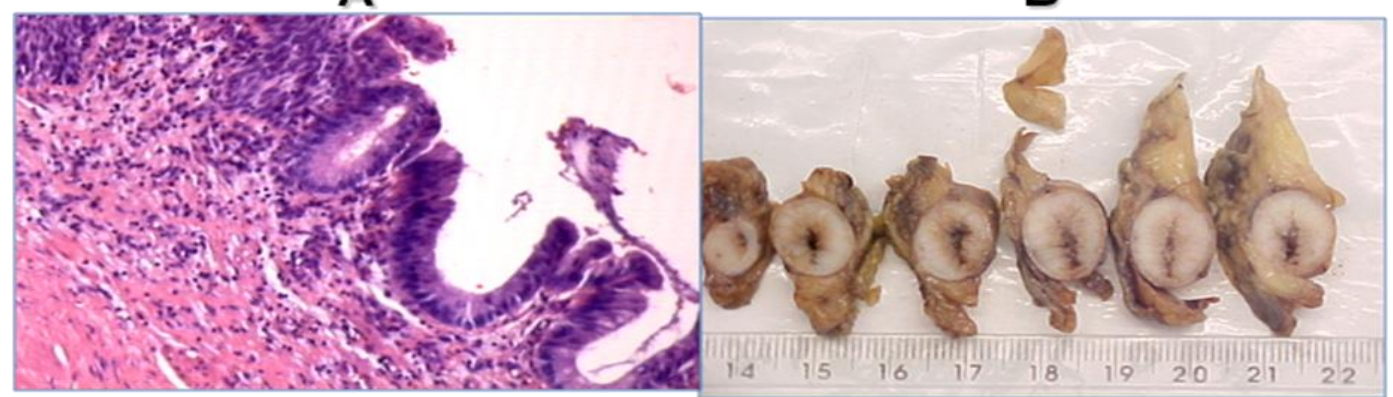

C

D 